\title{
Doktorarbeiten aus dem Medizinhistorischen Institut der Universität Basel 1969
}

I. Unter der Leitung von Prof. Dr.med.H.BuEss

a) Im Jahre 1969 gedruckte Dissertationen:

Eichenberger P., Johann Jakob Wepfer (1620-1695) als klinischer Praktiker, Basler Veröffentlichungen zur Geschichte der Medizin und der Biologie, Fasc. XXVI

Häfliger H., Zur Geschichte der Hämophilie, Basler Veröffentlichungen zur Geschichte der Medizin und der Biologie, Fasc. XXVIII

b) Im Druck befindlich:

KNüsel E., Über die Anfänge der pathologischen Anatomie und ihre Historiographie

c) Fertig, aber noch nicht gedruckt:

Tetzlaff D., Johannes von Miculicz und die Herausbildung der aseptischen Hilfsmittel

d) In Arbeit:

BRIN P., Joseph François Malgaigne, Kieferfrakturen

EISENRING, Regenerationsforschung im 19. Jahrhundert

Karahmehmedovic O., Ernst Tavel (1858-1912)

Moshfegh A.R., C.S.Haegler (1862-1916)

SсḦ̈R, Medizinalprüfungen in Basel

II. Unter der Leitung von PD Dr.H.M. Koelbing

a) Im Druck befindlich :

ZüLLIG S., Luigi Galvani (1737-1798), der Entdecker der Bioelektrizität

b) In Arbeit :

Kies V., Allag eines Engadiner Arztes um 1700

Schüler G., Friedrich Brenner (1809-1874)

KonfF R., Ärztliche Ethik bei Lorenz Heister (1687-1748)

III. Arbeit aus dem Medizinhistorischen Institut Basel

Grosch G., Dr. med., Falkenstein (Taunus), Johannes Wildberger (1815 bis 1879), Basler Veröffentlichungen zur Geschichte der Medizin und der Biologie, Fasc. XXVII. 